\title{
Graphene Applications in the Energy Field: State-of- the-Art and Impact
}

Juan Prieto Vivanco, MSc, ${ }^{1}$ and Carlos Rodríguez-Monroy, $\mathrm{PhD}^{2}$

${ }^{1}$ INDRA Corporation, Spain, jprietov@indra.es

${ }^{2}$ Universidad Politécnica de Madrid, Spain, crmonroy@etsii.upm.es

\begin{abstract}
Graphene with its unique properties combining mechanical resistance 200 times superior to steel, exceptional electrical, thermal, quantum and optical properties apart from an enormous versatility to modify its properties, has become a "technological catalyst" that allows the drastic evolution of a series of devices and systems existing until now limited in their capacities. At the same time, it opens new barely explored application fields related to nanomaterials science. Its mono atomic thickness and the need for precise control in its composition and structure to exploit its properties are posing a fundamental challenge for its manufacture and application, which is requiring enormous efforts by the scientific community and the industry. Without having yet achieved precise and complete control of the manufacturing chain, there are already small-scale graphene production chains exploiting different production methods and producing graphene worldwide to feed a first generation of graphene-based devices. The energy sector in a fundamental process of transformation towards the generation, and massive and sustainable supply of energy to supply an exponentially growing demand, is one of the sectors in which a more immediate impact of this first generation of systems based on graphene is foreseen. The imminent commercial appearance of a new generation of storage technologies based on graphene, will significantly break the technical-economic viability thresholds that were limiting applications such as mass storage of energy in electricity networks or transport applications. In subsequent waves (2020-2025), new cheaper and more efficient hydrogen and electric generation devices will increase the availability of cheap, sustainable and distributed energy. As can be seen from the increase in the number of patents in recent years in graphene-based technologies and the gradual emergence of commercial publications from the private sector, the market is in the commercial testing phase prior to commercial exploitation. Numerous private companies have been set up to capitalize on the value of this first generation of graphene commercial applications and large corporations and niche companies are accelerating their machinery to position themselves first in this market. The next wave of applications with substantially more disruptive impact is still in the conceptualization and proof of concept phase in laboratories and universities, requiring levels of technological maturity that are not expected before 2025.
\end{abstract}

Keywords- Graphene, Energy applications, Technological maturity

\section{INTRODUCTION}

Since its identification in 2004, graphene has become the 'holy grail' of materials science. Its electrical and mechanical properties have led to the opening of hundreds of research projects and development initiatives seeking to solve both its serial production and its application in fields as diverse as the development of high frequency electronic devices, advanced filters for desalination or neuronal interfaces. Among the multiple affected areas is the world of energy storage devices, with promises of new generations of batteries that can multiply by 10 the power density of current lithium ion batteries with load times of the order of few hours, super capacitors or new cheaper photovoltaic cells with better use of solar energy. If these projections were to be fulfiled, the world of energy, transport and electronic devices with autonomous power supply, would be radically transformed. Despite the enthusiasm in the scientific community, it is not yet clear what will be the real impact of graphene in this domain or what the real implications of this application would be for the different markets.

\section{GRAPHENE PRODUCTION. TECHNICAL AND ECONOMIC STUDY}

The properties of Graphene vary drastically depending on the purity, size and shape of the crystals and the way in which the graphene sheets are arranged with each other and with other materials. Graphene series production is economically the great challenge that once solved will allow the development of the hundreds of applications.

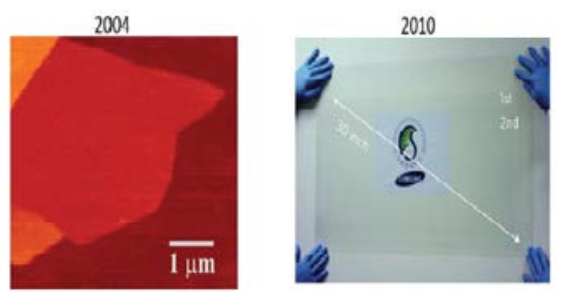

Fig. 1: Evolution in the size of the graphene crystals produced, from 1micrometer graphene flakes produced by exfoliation to reel processing [1]

At present, there are different methods of graphene production, each with different costs, degrees of purity, crystal sizes and chemical modifications that fundamentally condition its application. At present, 8 basic blocks of processes for the synthesis of graphene and its derivatives are distinguished. Despite the progress made in recent years in the production of graphene, the production of large graphene crystals of monatomic uniform thickness on any type of substrate remains a challenge in the production of graphene, as is the cost of production. 


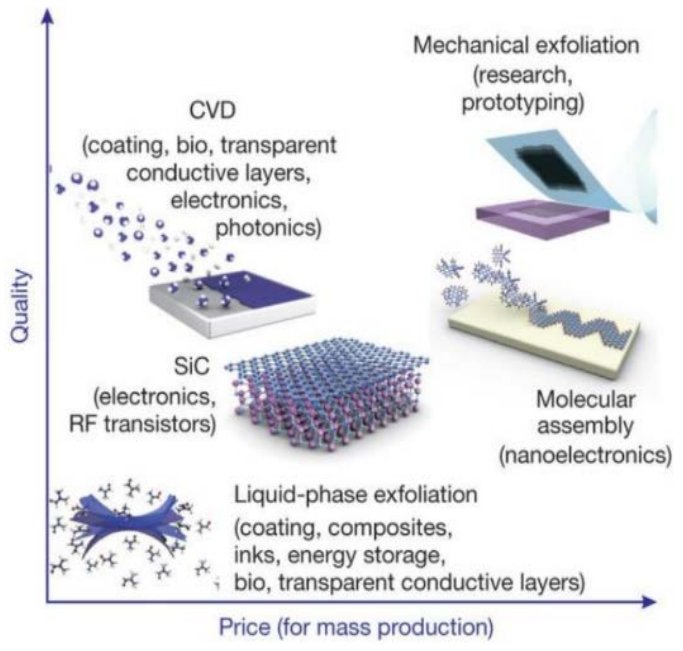

Fig. 2: Relative price and quality of graphene depending on the production method [1]

Given the different qualities, production efficiency and substrates, of the different synthesis methods identified to date, the following methods have been identified as the most promising for the production of graphene for energy applications:

- Liquid phase exfoliation.

- Chemical vapor deposition.

- Growth on silicon carbide.

\section{THE APPLICATION OF GRAPHENE TO ENERGY STORAGE AND PRODUCTION}

\section{A. Current reality and challenges of the energy sector}

Production / conversion, transport and storage are the essential functions of any energy system. Electricity due to its versatility in the conversion to and from other energy sources and its ease of transportation has become the main energy vector of our society, being at the base of almost all sectors of activity. Hydrocarbons in the form of gas and petroleum derivatives are other energy vectors with great development and wide use throughout the planet, mainly for thermal uses and transportation. The abundant and cheap availability of these two energetic vectors has constituted the base of the industrial revolution, and the technological revolution that our society has experienced in the last three centuries.

Problems associated with sustainability over time and the availability of resources force the energy sector to address three major, intimately related fundamental challenges: a. Massive reduction of emissions of carbon dioxide to the atmosphere, forcing the substitution of energy sources based on fossil fuels for other energy sources that do not emit greenhouse gases.

b. Efficient storage. The presence of non-manageable energy sources in systems that depend on a continuous and reliable supply over time, as well as the need to replace fossil fuels as a source of energy storage for transport, require storage and storage elements, in order to convert rapidly large volumes of energy either in a fixed or in a mobile way (electric vehicles and other mobile devices).

c. Maintain the supply of increasing amounts of energy cheaply, that sustain the growing energy needs of a world population increasingly developed industrially and with growing energy needs.

The transformation of the energy sector is being supported on technological storage and generation solutions that after a very intense development during the last decades begin to show signs of depletion, facing three basic challenges:

1. Efficiency increase in energy storage and conversion processes.

2. Reduction of production costs.

3. Decrease in size / weight of the conversion and storage devices in relation to the storage capacity / energy conversion, reducing the needs of materials and favoring their mobility.

The Ragone diagram allows the different energy storage / conversion devices to be classified according to their peak power and their energy storage capacity per unit mass.

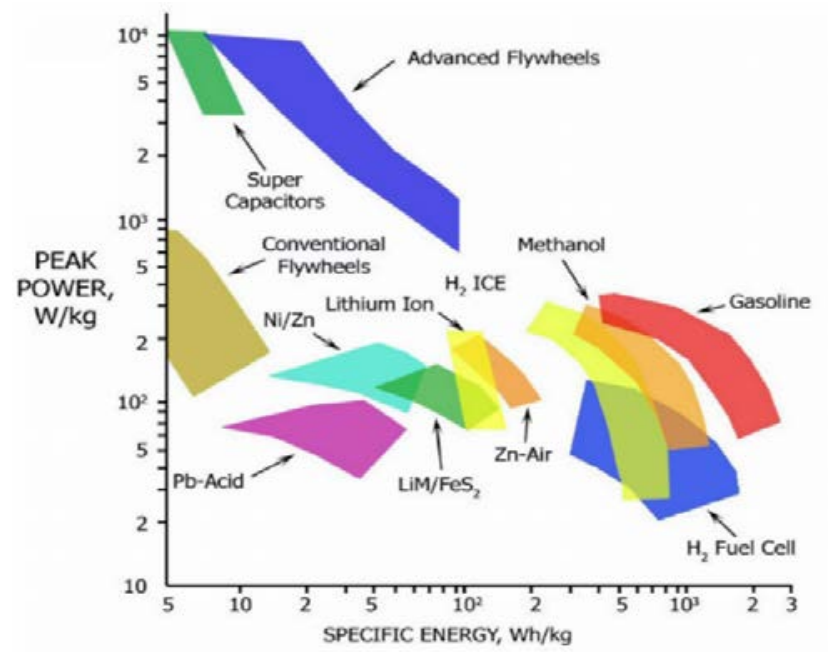

Fig. 3: Ragone diagram corresponding to the different commercial energy storage technologies

Graphene for its exceptional electrical, optical and physical properties, and abundance and low cost of its raw 
material, has become the focus of the main groups of research and marketing of energy storage and conversion solutions. In the following sections, in order to understand their potential impact, graphene applications are analyzed for storage and conversion of energy that present a higher degree of maturity.

\section{B. Storage devices: batteries and super capacitors.}

\section{Challenges}

The use of batteries and super capacitors as energy storage devices faces three fundamental challenges already identified in the general introduction of the previous section:

1. Increased efficiency in storage processes: minimization of losses in the charging / discharging process and maintenance during the life of the battery, measured in the form of number of charge and discharge cycles.

2. Reduction of production costs. Substituting raw materials and high cost production processes for other cheaper materials and processes.

3. Increase in storage density and power, increasing the power and maximum power of the battery per unit mass.

The electrochemical battery is the most widespread storage mechanism by degree of maturity and allows higher storage densities at the lowest cost. The capacitors, unlike the batteries, are characterized by their fast loading and unloading power, but also by their low energy storage capacity and short retention times of stored energy. The density of storage and power density of electrochemical batteries has been increasing, being Lithium ion batteries that achieve maximum energy density and power per unit mass and volume. The specific densities reached by this type of batteries have allowed the commercial development of electric vehicles, evolving from the initial $150 \mathrm{~km}(100 \mathrm{Wh} / \mathrm{kg})$ of autonomy to $400-500 \mathrm{~km}$ ( $250 \mathrm{Wh} / \mathrm{kg}$ ) of the new generation.

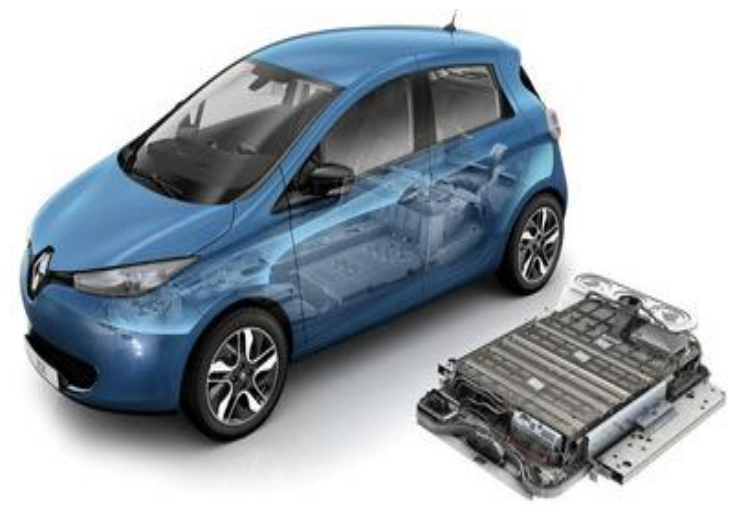

Fig.4: Lithium-nickel-manganese-cobalt battery systems of the Renault $\mathrm{ZOE}$ with a range of $403 \mathrm{~km}$
Although the peak powers of these batteries are comparable to those of combustion engines, the power and energy density per unit mass is still significantly below. This limitation in the power density leads to excessively long load times of close to 40 hours for $400 \mathrm{~km}$ of battery life with a normal $220 \mathrm{~V}$ socket. The capacity of current lithium-ion batteries and super capacitors are fundamentally limited in performance, loading and unloading speed and number of cycles for the chemical and physical properties of the materials used in the anodes and cathodes. The loading and unloading processes produce physical and chemical variations in the electrodes, which increase with the speed of the process as the temperature increases and the number of cycles increases. Table 1 summarizes the current situation of storage solutions in terms of energy density and cost per unit of energy and the target values for the different applications.

TABLE I

CURRENT VALUES AND FUTURE TARGETS FOR THE APPLICATION OF BATTERIES TO DIFFERENT ENERGY DOMAINS

\begin{tabular}{c|c|c|c|}
\multicolumn{1}{c}{} & Current & \multicolumn{2}{c}{ Target Values } \\
\cline { 3 - 4 } & values & Transport & Electric \\
& & & Grid \\
\hline Specific Energy $(\mathrm{Wh} / \mathrm{kg})$ & $250-250$ & $600-1000$ & - \\
\hline Cost $(\$ / \mathrm{kWh})$ & 250 & 100 & 100 \\
\hline
\end{tabular}

Application of graphene.

Graphene, due to its high surface / volume ratio, high load mobility, physical stability and chemical stability, allows the electrodes to increase the specific power of the batteries, increasing the charge / discharge speed and the number of cycles the battery is capable to endure. For hybrid lithiumsulfur (Li-S) batteries and architectural cathodes hybridized with sulfur, graphene and carbon nanotubes, energy densities of up to $400 \mathrm{Wh} / \mathrm{kg}$ and power of $10,000 \mathrm{~W} / \mathrm{kg}$ are estimated [80].
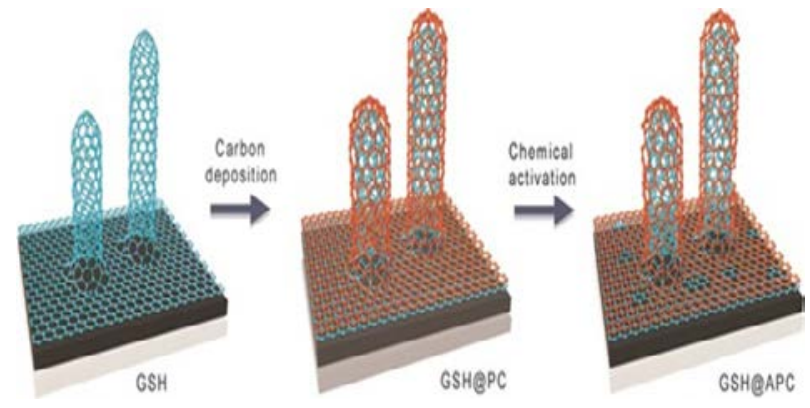

Fig. 5: Figure of hybrid structures Graphene-carbon nanotubes GSH@APC [2]

16 $^{\text {th }}$ LACCEI International Multi-Conference for Engineering, Education, and Technology: "Innovation in Education and Inclusion”, 19-21 July 2018, Lima, Peru. 
Fig. 6 shows on a Ragone diagram, the results obtained in some experimental prototypes.

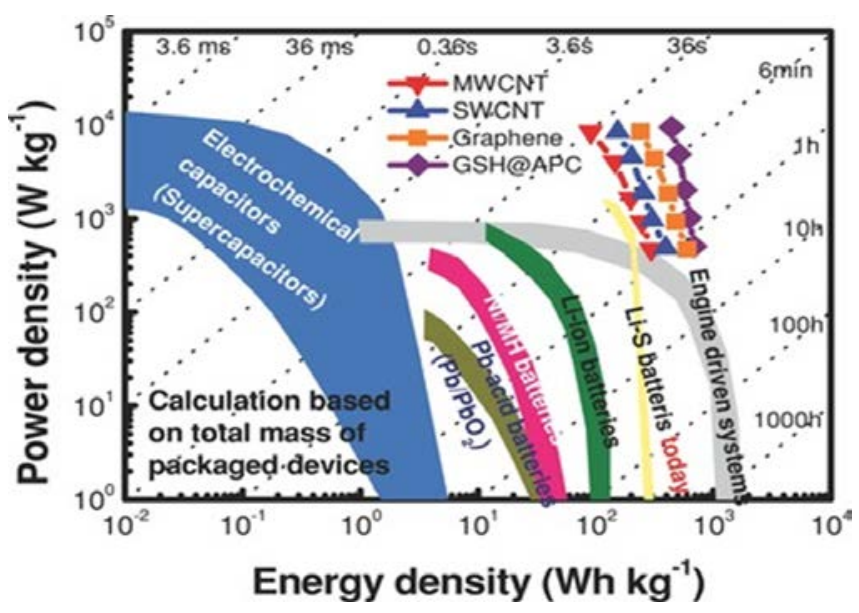

Fig. 6: Ragone diagram for Li-S batteries based on different cathodes based on hybrid architectures of graphene and carbon nanotubes (and comparison with other storage technologies [2]

Another very interesting application is the hybrid storage systems (Battery-supercapacitor hybrid device BSH) in which a battery anode is combined with a super capacitor cathode, combining the very high power density of the super capacitors with the high energy density of Battery. BSH prototypes of Ion Sodium with graphene anode have achieved power densities of 16,500 W/ kg and energy densities of $285 \mathrm{Wh} /$ $\mathrm{kg}$ [3].

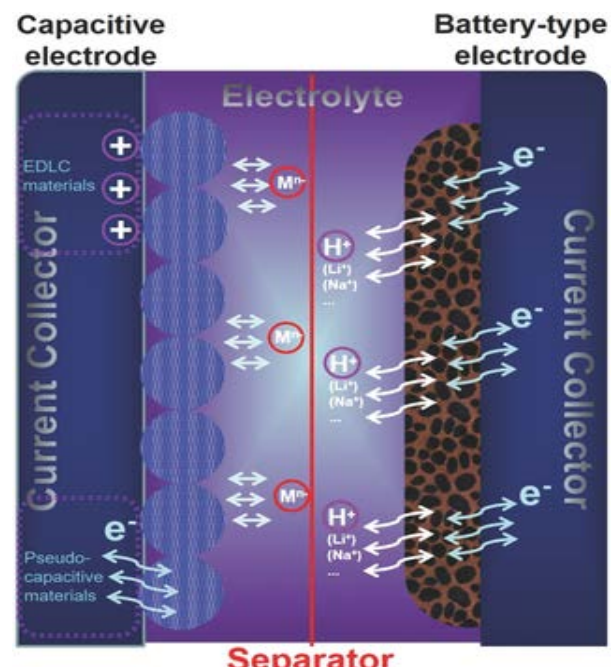

Fig. 7: Scheme of hybrid super capacitor storage battery system [3]

The degree of technological maturity is measured by the technology readiness level (TRL). In the case of batteries and super capacitators this level is TRL8.

\section{Existing prototypes.}

There are already prototypes that substantially exceed the performance of current technologies. Currently, they are in the reduction of costs and commercial production stage and an imminent appearance of commercial products is expected. There are some external mobile batteries on the market that integrate graphene into the electrodes, tripling the charging and discharging speed of standard Li-ion batteries.

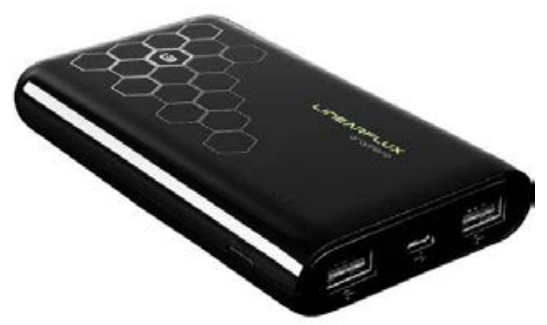

Fig. 8: Commercial lithium ion mobile charging battery and graphene electrodes, allowing 3 times faster charges and downloads

Samsung SID reports in 2017 on its website the manufacture of a new generation of lithium-ion batteries with "beads" of graphene (GB) in cathodes and anodes, allowing a $45 \%$ increase in the capacity of the batteries $(444 \mathrm{Wh} / \mathrm{kg}$ at $60^{\circ} \mathrm{C} 800 \mathrm{Wh} / \mathrm{l}$ ) and charging and discharging speeds 5 times higher, operating at temperatures of $60^{\circ} \mathrm{C}$ which reduces the cooling needs.

\section{Fuel cells and storage of hydrogen.}

\section{Challenges}

Fuel cells convert the chemical energy stored in a fuel into electricity through its oxidation. The electrochemical process is direct without thermal conversion processes, intermediate mechanics, achieving efficiencies in the conversion of fuel energy much higher than that of any 40$60 \%$ conversion engine, and can reach $85-90 \%$ with cogeneration (taking advantage of the residual heat). The main problem of the current fuel cells in their different configurations is the high production cost associated with the need to use noble metals as catalysts of the oxidation reaction ( $\mathrm{Pt}, \mathrm{Au}, \mathrm{Ru})$. Currently the cost of fuel cells is around \$ 53 / $\mathrm{kW}$ and the objective to make them competitive is to reduce it to $\$ 30 / \mathrm{kW}$ [DOE 12].

Other challenges faced by fuel cells are:

- Temperature management. Maintenance of constant temperature throughout the cell to avoid its degradation.

- Useful life and other specific requirements related to the range of operating temperatures.

- Limited tolerance to CO (carbon monoxide). 
For fuel cell applications related to the use of hydrogen as fuel (automotive industry), other challenges arise related to the economic production and safe and efficient storage of hydrogen, which in gaseous form occupies volumes too high for automotive applications.

\section{Application of graphene}

The high surface ratio, excellent thermal conductivity, mechanical stability and electronic mobility make graphene an ideal candidate to replace the current catalysts based on noble metals of high cost $(\mathrm{Pt}, \mathrm{Au}, \mathrm{Rb})$ in anodes and cathodes of fuel cells allowing the increase of efficiency, power density, stability and cost reduction. This catalyst function is maximized by favoring the catalizing activity of graphene edges, achieving improvements of $70 \%$ with respect to $\mathrm{Pt}$ catalysts [4].

Additionally, in fuel cells designed with an exchange membrane, graphene in combination with Boron Nitride, forms membranes highly permeable to the passage of protons and impervious to the passage of any other chemical species, thus avoiding contaminations that significantly reduce the efficiency of current fuel cells.

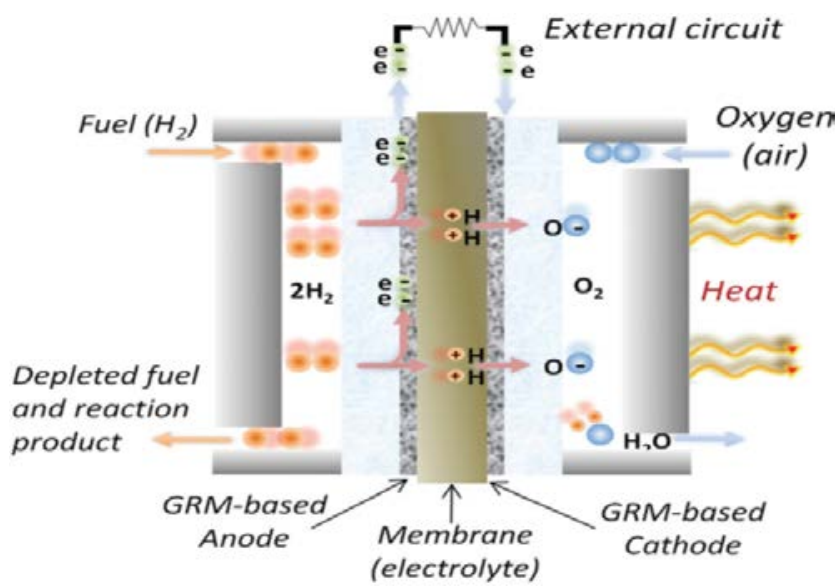

Fig 9: Proton exchange membrane fuel cell. Graphene acts as a catalyst separating $\mathrm{H}_{2}$ fuel in hydrogen cations and electrons, to combine them later in the cathode with oxygen, generating water, heat and electric current [4].

Additionally, other applications related to the hydrogen chain are being studied:

1. Hydrogen storage. Forms derived from graphene allow the absorption and controlled release of hydrogen, allowing its compact and safe storage.

2. Generation of hydrogen from solar energy. The permeability of graphene membranes to the passage of protons could be used to produce hydrogen from water vapor molecules at much lower costs than the current hydrolysis methods.

Current degree of technological maturity: TRL 4-5
Individual prototypes already overcome the properties of membranes and catalysts currently in the market. Research is being done on the combination of these various functions in complete commercial fuel cell prototypes.

\section{Photovoltaic production based on graphene}

\section{Challenges}

The direct production of electrical energy from solar radiation, being an area with a high degree of maturity, faces two fundamental challenges:

1. The increase in efficiency in the conversion of solar energy into electrical energy currently around $25 \%$ in crystalline silicon panels.

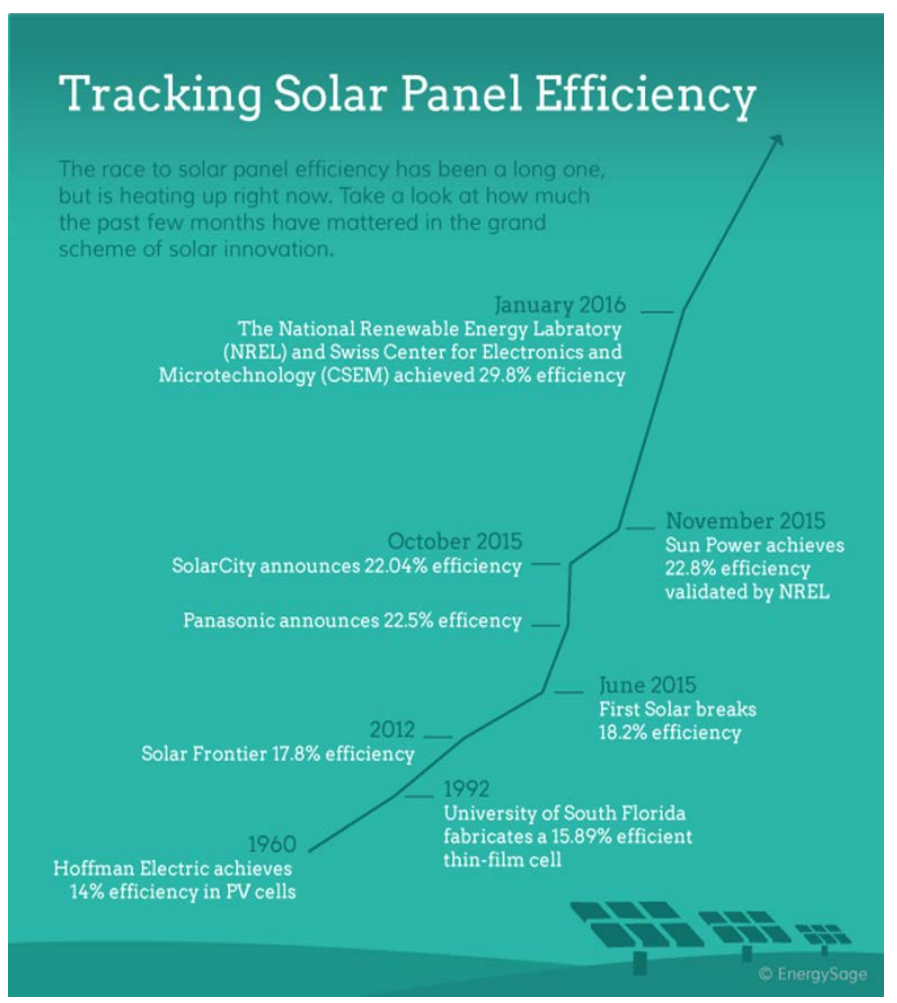

Fig. 10: Evolution of the efficiency in the conversion of solar into electricity [5]

2. The reduction of the cost of production. In continuous process of reduction by optimizations in the manufacturing process, but slowing down in the last ones, due to the cost of the materials. 


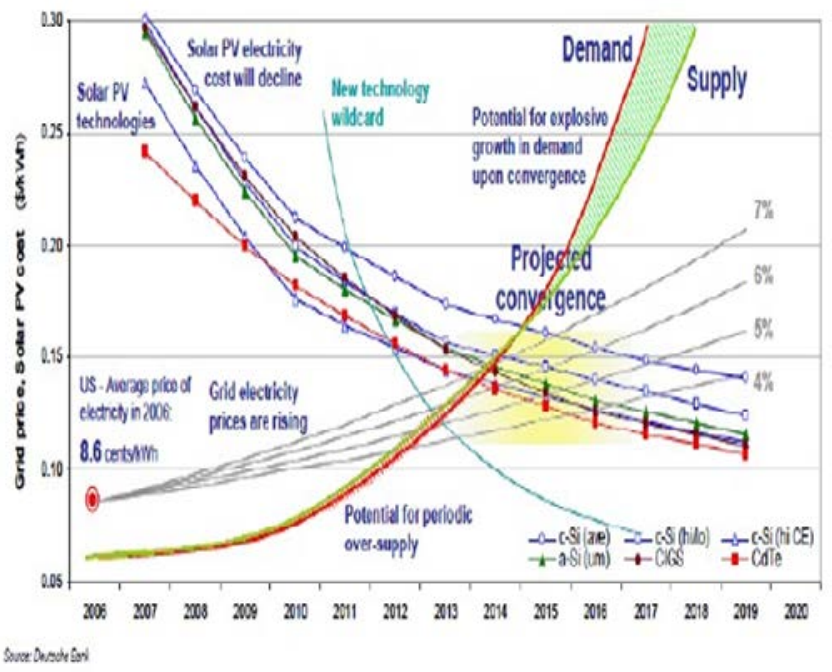

Fig. 11: Evolution of the cost of solar generation compared to the cost of electricity supply [13]

Most of the photovoltaic cells manufactured are based on silicon, its performance in the conversion being conditioned to the purity of the silicon and the degree of alignment of its silicon molecules. Based on these parameters, three fundamental types of photovoltaic cells can be identified:

- Monocrystalline Silicon (Mono-Si). Unique Si crystals of high purity, high performance in the conversion of energy between 15-25\%, and maximum useful life. They are substantially more expensive and more susceptible to damage by snow and dust than other types.

- Polycrystalline silicon (p-Si / mc-Si). Simpler treatments and lower purity silicon is used, reducing the final cost of production. This reduces its performance to $13-16 \%$.

- Thin Film Cells (Thin Film Solar Cells, TFSC). They are manufactured by deposition of thin layers of photovoltaic material on a substrate. This type of cells achieve yields between 7 and 13\%, are easy for mass production, flexible and potentially cheaper than crystalline $\mathrm{Si}$. They degrade more quickly than those of crystalline $\mathrm{Si}$.

\section{Application of graphene}

The high transparency of graphene to incident light throughout the frequency range, together with its mechanical and electrical properties, make graphene an ideal material to perform different functions in photovoltaic cells:

1. Photo anodes for their efficiency in the transport of charges, preventing recombinations.

2. Photosensitive element producing electrons under irradiation in a greater frequency range,

3. Structural material, providing flexibility and resistance to the photovoltaic cell while maximizing the range of usable light frequencies.
The application of graphene to photovoltaic production should allow:

- To reduce costs by minimizing the use of noble metals such as platinum.

- To increase the mechanical strength and flexibility of the panels increasing the range of uses.

- To increase the performance in the conversion of light above the current $25 \%$.

The most promising results have been obtained using graphene in a new generation of solar cells based on Perovskite (PSCs), which has aroused great interest in the rapid evolution in the conversion performance of sunlight (3.8\% in 2009 to $22.1 \%$ in 2016 as shown in Figure 12) and its low production cost and compatibility with flexible plastic substrates.

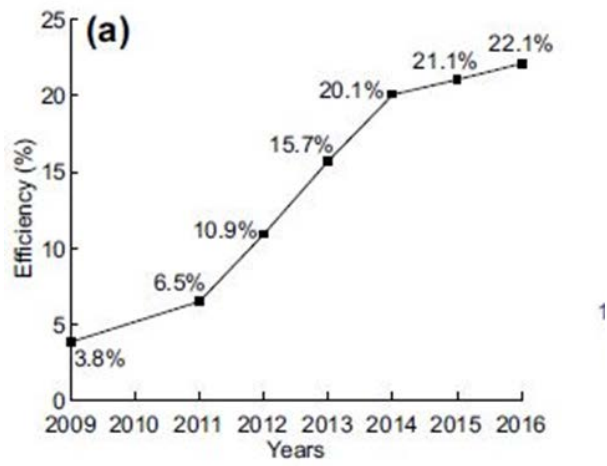

Fig. 12: Performance evolution of Perovskite-based cells (PSC). Yields above $18 \%$ have been obtained using graphene as a conductive electrode [6]

Current degree of technological maturity: TRL 4-5

There are already PSC and graphene cells that have equivalent yields to those of monocrystalline $\mathrm{Si}$ at a theoretically lower cost. It is necessary to optimize designs and serial production processes.

\section{F. Impact study of graphene on different energy storage sectors}

As identified in the previous section, a gradual arrival of commercial applications of graphene in the energy field is expected:

1. Imminent 2018-2019. First wave of applications of graphene with the first commercial models of batteries and super capacitors.

2. 2019-2020. Second wave with the first commercial models of battery hybrids with super-capacitor cathodes and photovoltaic solar cells that match the performance of current silicon crystalline cells significantly reducing the cost. 
3. 2021-2025. Third wave with the arrival of the second generation of low cost hydrogen cells replacing noble metal catalysts by graphene and hybrid battery systems with super capacitors in the cathode.

4.> 2025. Commercial application of other more disruptive technologies such as a third generation of photovoltaic cells with yields higher than 30\% (based on complex architectures of uncertain graphene), H2 storage in graphene structures, thermoelectric generation devices for energy collection and nanogenerators, in a new generation of self-supplied electric devices.

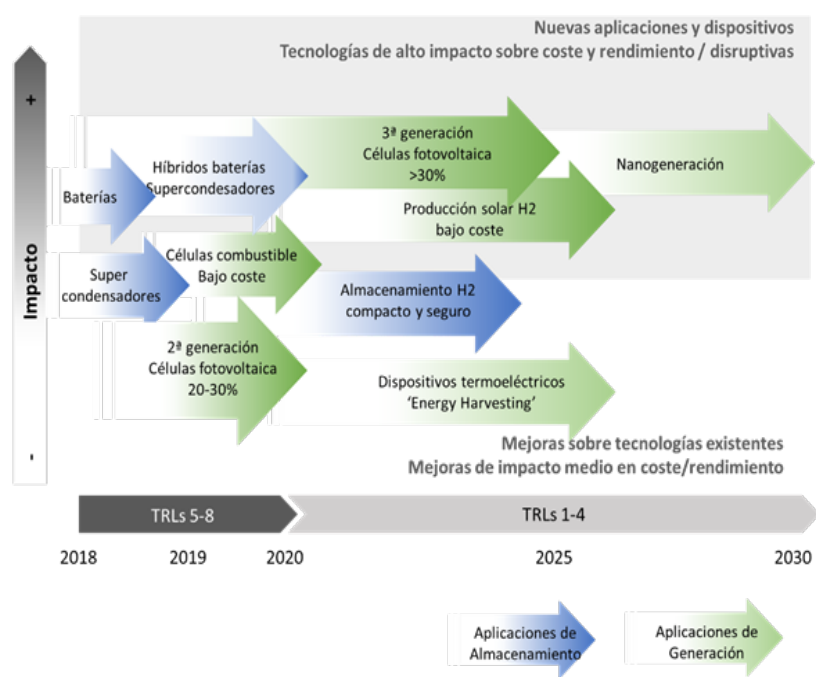

Fig. 13: Applications of graphene in the energy field: Impact and roadmap

G. Analysis of national and international markets.

Raw material and manufacturing capacity. One of the first elements in the market analysis is the consideration of the distribution of the raw material graphite in the world and the current production capacity of graphene. In Figure 14, we can see the distribution of global reserves of graphite in the world.

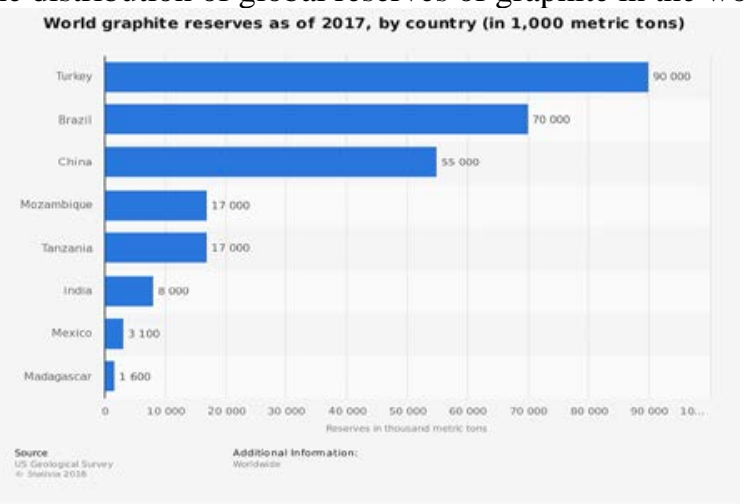

Fig. 14: Global reserves of graphite in 2017 [7]

The main volume of reserves is concentrated in Turkey, Brazil, China and Mozambique. This concentration of reserves can be a problem when creating an open market for the supply of graphite for the top-down manufacture of graphene, and it is a clear competitive advantage for China, one of the most active countries in the research of the applications of graphene. As identified in Section 2, there are, apart from manufacturing methods from graphite, other methods that start from other carbon sources, such as hydrocarbons (ethane, methane, etc.). This opens up other alternatives for the production of graphene that does not require the use of graphite. The following figures show the distribution of graphene production, by country, still limited and how this production is shared between top-down methods (72\%) from graphite and bottom-up methods from other carbon sources (28\%).

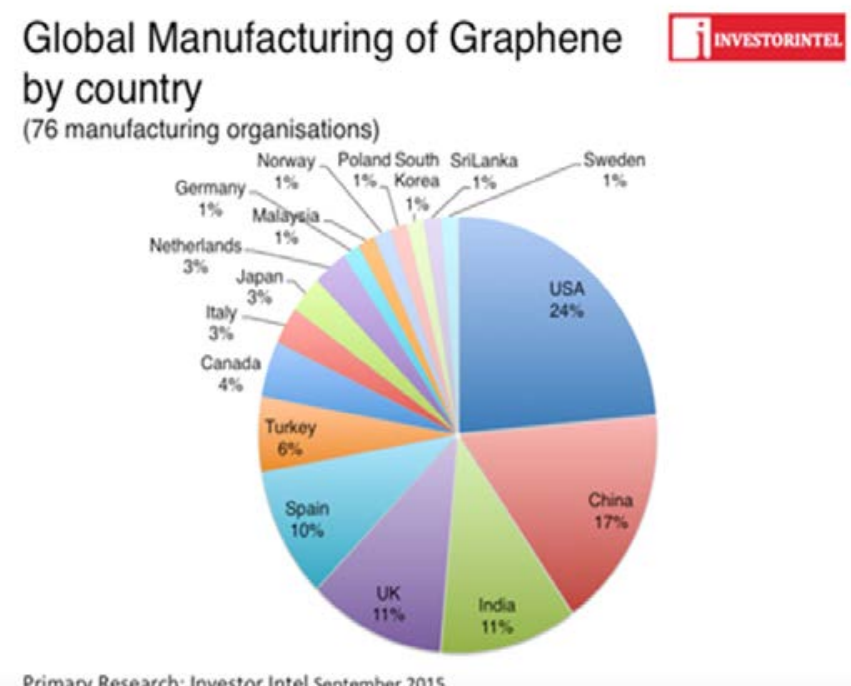

Fig. 15: Global distribution of graphene production in 2015 [8]

\section{Global Manufacturing of Graphene jimvstonsma in 2015 \\ (76 manufacturing organisations)}

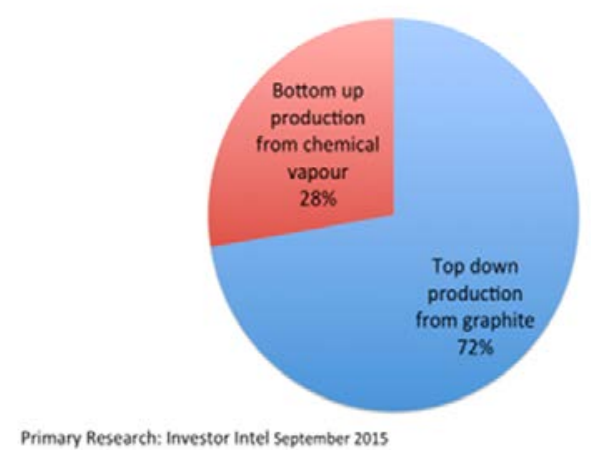

Figure 16: Distribution of global production of graphene based on the raw material of origin in 2015 [8]. 
Research efforts and proximity of marketing.

Since the discovery of graphene in 1986, the number of published academic articles related to graphene has grown exponentially. This is a direct measure of the enormous interest that graphene is raising around the world, as well as the resources that are turning to make the technological application of graphene a reality in the different areas of potential application.

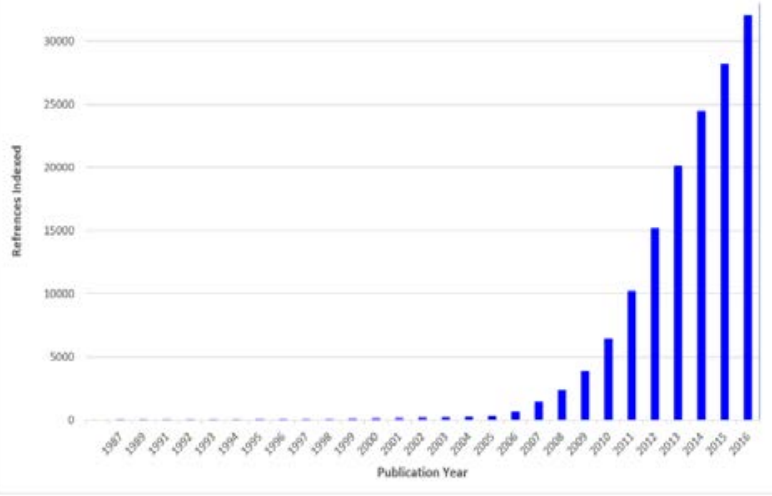

Fig. 17: Evolution of the number of annual publications related to Graphene (SciFinder Credit: Joseph Meany) [9].

As has been confirmed by the advanced maturity level of the first waves of commercial applications of graphene, the interest has transcended in recent years the academic field, and has spread throughout the world in the form of patents, annually triggering the number of patents of companies and technological institutions which could indicate the imminent appearance of commercial applications of graphene in the next two years. If we analyze the distribution of patents by companies / institutions, the number of patents registered by Samsung and universities in Korea and China stands out.

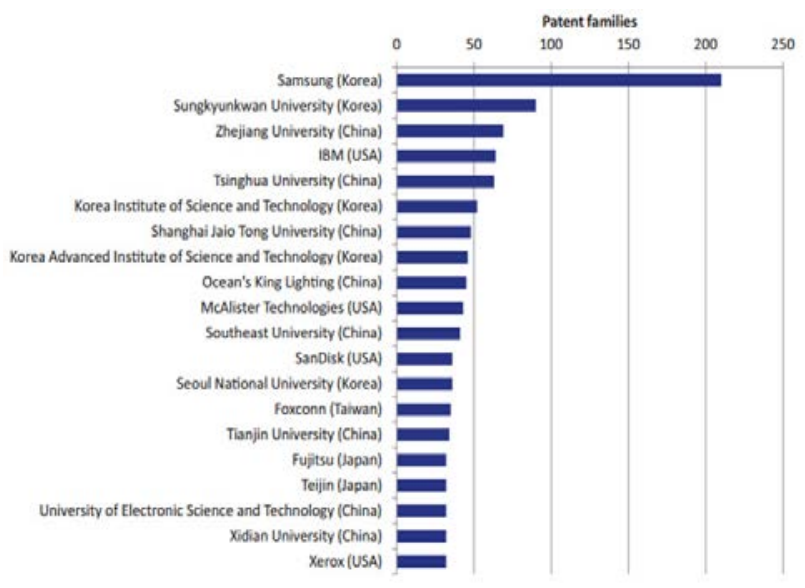

Fig. 18: Ranking of the companies with the largest number of patents related to graphene [10]
Analysts expect an exponential growth of global graphene demand from the current \$ 40 million to \$ 120 million in 2020 , representing uses for energy approximately $30 \%$ of total demand by 2020 [11].

\section{Total Graphene Market will Reach $\$ 126$ million in 2020}

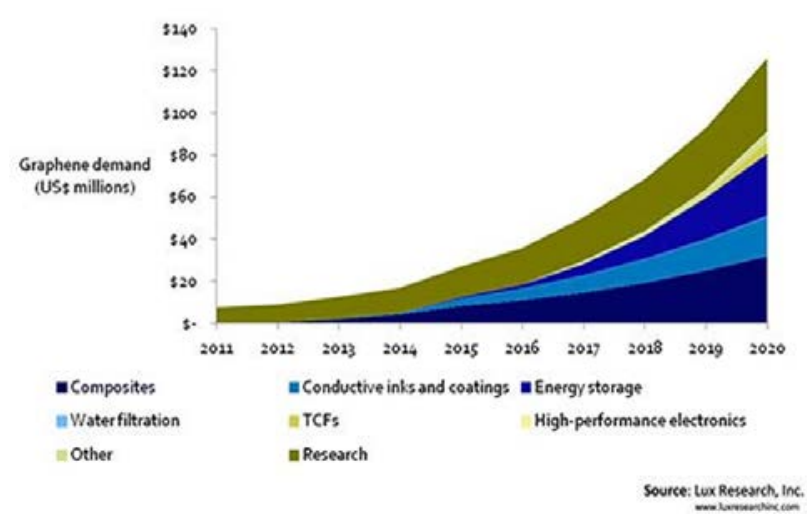

Fig. 18: Ranking of the companies with the largest number of patents related to graphene [10]

\section{CONCLUSIONS}

Graphene in its different forms with its unique properties combining mechanical strength 200 times superior to steel, exceptional electrical, thermal, quantum and optical properties apart from an enormous versatility to modify its properties by functionalization with other chemical species or other materials through the formation of composite materials, it has become a "technological catalyst" that allows the drastic evolution of a series of devices and systems existing until now limited in their capacities while opening new fields of application hardly explored related to the science of nanomaterials. Its mono-atomic thickness and the need for precise control in its composition and structure to exploit its properties are posing a fundamental challenge for its manufacture and application, which is requiring enormous efforts by the scientific community and the industry.

Without having yet achieved precise and complete control of the manufacturing chain, there are already small-scale graphene production chains exploiting different production methods and producing graphene worldwide to feed a first generation of graphene-based devices. This first generation of devices, imminent in the different markets (2018-2023) focuses on improving existing technologies of high demand, combining graphene with other materials to improve their performance, their operating ranges and reduce their costs. The next generations of graphene applications require more fundamental knowledge and exploitation of graphene and the development of theories and architectures of materials barely

16 $^{\text {th }}$ LACCEI International Multi-Conference for Engineering, Education, and Technology: "Innovation in Education and Inclusion”, 19-21 July 2018, Lima, Peru. 
explored to date that involve the opening of new fields of application disruptive to current technology.

The energy sector in a fundamental process of transformation towards the generation, and massive and sustainable supply of energy to supply an exponentially growing demand, is one of the sectors in which a more immediate impact of this first generation of systems based on graphene is foreseen. The imminent commercial appearance of a new generation of storage technologies based on graphene, will significantly break the technical-economic viability thresholds that were limiting applications such as mass storage of energy in electricity networks or transport applications. In subsequent waves (2020-2025), new cheaper and more efficient hydrogen and electric generation devices will increase the availability of cheap, sustainable and distributed energy.

As can be seen from the trickle in the number of patents in recent years in graphene-based technologies and the gradual emergence of commercial publications from the private sector, the market is in the commercial testing phase prior to commercial exploitation. Numerous private companies have been set up to capitalize on the value of this first generation of graphene commercial applications and large corporations and niche companies are accelerating their machinery to position themselves first in this market. The next wave of applications with substantially more disruptive impact is still in the conceptualization and proof of concept phase in laboratories and universities, requiring levels of technological maturity that are not expected before 2025.

This profound technological transformation in successive waves should not be attributed exclusively to graphene. Graphene and its derivative forms, due to their exceptional properties and based in widely accessible carbon, have acted as a technological catalyst, forcing the scientific community and technology companies to focus their attention on the 'nano' scale, opening the door to engineering of new materials and devices with completely new macroscopic properties built to measure from a fundamental knowledge of the physics behind the interactions between atoms.

\section{REFERENCES}

[1] Ferrari, Andrea C. et al. "Science and Technology Roadmap For Graphene, Related Two-Dimensional Crystals, And Hybrid Systems." Nanoscale, vol 7, no. 11, 2015, pp. 4598-4810. Royal Society Of Chemistry (RSC), doi:10.1039/c4nr01600a.

[2] Peng, Hong-Jie \& Huang, Jia-Qi \& Zhao, Meng-Qiang \& Zhang, Qiang \& Cheng, Xin-Bing \& Liu, Xin-Yan \& Qian, W.Z. \& Wei, Fei. (2014). Carbon: Nanoarchitectured Graphene/CNT@Porous Carbon with Extraordinary Electrical Conductivity and Interconnected Micro/Mesopores for Lithium-Sulfur Batteries (Adv. Funct. Mater. 19/2014). Advanced Functional Materials. 24. . 10.1002/adfm.201303296.
[3] Zuo, Wenhua, et al. "Battery-Supercapacitor Hybrid Devices: Recent Progress and Future Prospects.” Advanced Science, vol. 4, no. 7, 2017, p. 1600539., doi:10.1002/advs.201600539

[4] "Platinum meets its match in quantum dots from coal." Rice News, news.rice.edu/2014/10/01/platinum-meets-its-match-in-quantum-dotsfrom-coal/.

[5] Matasci, Sara. "How Solar Panel Cost \& Efficiency Have Changed Over Time | EnergySage.” EnergySage Solar News Feed, EnergySage, 24 Jan. 2018, news.energysage.com/solar-panel-efficiency-cost-over-time/.

[6] Lim, Eng Liang, et al. "A Mini Review: Can Graphene Be a Novel Material for Perovskite Solar Cell Applications?” Nano-Micro Letters, vol. 10, no. 2, 2017, doi:10.1007/s40820-017-0182-0.

[7] "World graphite reserves top countries 2017 | Statistic." Statista, www.statista.com/statistics/267367/reserves-of-graphite-by-country/.

[8] "Who is making graphene, and where - Examining a secretive market." InvestorIntel, 22 Oct. 2015, investorintel.com/sectors/technologymetals/technology-metals-intel/who-is-making-graphene-and-whereexamining-a-secretive-market/.

[9] Js, Ahn S Sung, and Kim Hj Sung Yk. "Emerging Analysis on the Preparation and Application of Graphene by Bibliometry.” Journal of Material Science \& Engineering, vol. 04, no. 05, 2015, doi:10.4172/21690022.1000192

[10]Sebastian Anthony on May 19, 2014 at 2:34 pm Comment. "The next Apple-Samsung battle will be fought over graphene, not in the courtroom." $\quad$ ExtremeTech, 19 May 2014, www.extremetech.com/computing/182657-the-next-apple-samsungbattle-will-be-fought-over-graphene-not-in-the-courtroom.

[11]Harold Goldmeier Growth, momentum, growth at reasonable pricelinkden.cls-1\{fill:\#024999;\}. “Graphene Investing: 2018.” Seeking Alpha, 16 Jan. 2018, seekingalpha.com/article/4137774-grapheneinvesting-2018. 